\title{
Évolution de la croissance radiale du hêtre (Fagus silvatica $L$ ) dans les Vosges. Premiers résultats sur le versant lorrain
}

\author{
JF Picard \\ INRA-Nancy, unité d'écophysiologie forestière, équipe Phyto-écologie, 54280 Champenoux, France
}

(Reçu le 25 octobre 1993; accepté le 18 février 1994)

\begin{abstract}
Résumé - À la suite des travaux sur le dépérissement du sapin (Abies alba Mill) dans les Vosges et de ceux sur le hêtre (Fagus sylvatica $L$ ) dans la plaine lorraine, et en raison de résultats apparemment contradictoires sur ces 2 espèces, on a entrepris une étude dendroécologique des hêtres de la hêtraie sapinière des Vosges. Cet article fait le point des résultats obtenus pour les 537 arbres des 85 premières placettes échantillonnées sur le versant lorrain des Vosges. Par rapport au sapin, et dans la même région, on observe un certain nombre de convergences de comportement, mais aussi des différences : i) certaines années caractéristiques sont communes, mais pas toutes ; les cernes manquants sont moins fréquents, et rarement successifs ; ii) la courbe d'accroissement moyen en fonction de l'âge atteint son maximum plus tard que chez le sapin (vers 40 ans au lieu de 10 ans) iii) pour un âge donné, la largeur des cernes du hêtre augmente de façon notable avec la date (ils sont d'autant plus larges qu'ils ont été élaborés à une date récente) ; iv) comme pour le sapin, mais contrairement au hêtre de la plaine lorraine, on observe une nette tendance à long terme à l'augmentation de la largeur des cernes, de l'ordre de $70 \%$ entre 1850 et 1989 , avec des périodes de crise qui interviennent approximativement aux mêmes périodes ; en revanche, il semble que l'augmentation de la largeur des cernes ait été plus régulière que pour le sapin : on n'observe pas de palier aussi net que pour le sapin au milieu du $x x^{\mathrm{e}}$ siècle. On conclut que les différences de comportement entre sapin et hêtre sont très probablement dues à un effet espèce, sans exclure l'effet des différents paramètres du milieu : c'est la suite de l'étude, lorsque l'ensemble du massif vosgien aura été prospecté, qui permettra de faire la part des 2 effets ainsi que d'expliciter les causes écologiques des différents phénomènes mis en évidence dans cette première étude.
\end{abstract}

dendrochronologie / dendroécologie / croissance radiale / années caractéristiques / hêtre / Vosges

Summary - Changes in radial growth of beech (Fagus sylvatica $L$ ) in the Vosges. Preliminary results from the Lorraine catchment area. Following work on the decline of silver fir (Abies alba Mill) in the Vosges and beech (Fagus sylvatica $L$ ) in the Lorraine plain, which seemed to show contradictory results, a dendrochronological study of beech from mixed beech/fir stands in the Vosges was carried out. This paper presents the results obtained for 537 trees from the first 85 sites sampled on the 
Lorraine side of the Vosges. A certain number of behavioural similarities have been observed for spruce in the same area and also some differences: i) certain pointer years are common to both species but not all; missing annual rings are less frequent and rarely successive; ii) the mean growth curve as a function of age reaches a maximum later than that of fir (at about 40 years old instead of 10 years); iii) for a given age, the increase of annual ring width for beech is highly correlated to date (the more recent rings are relatively larger); iv) as for spruce, but contrary to beech in the Lorraine plain, a strong tendency to a long-term increase in ring width was observed. This was in the order of $70 \%$ between 1850 and 1989, with crisis periods, which always occurred at about the same time. Conversely, the ring-width increase occurred more regularly than in fir and the plateau in the middle of the 20 th century is not as clear as that of the fir. In conclusion, the behavioural differences between fir and beech are probably due to a species effect, but environmental differences should be ruled out. The next stage of this study, in which the whole of the Vosges area will be surveyed, will allow us to separate the 2 factors, as well as explain the ecological causes of the different phenomena identified in this initial study.

dendrochronology / dendroecology / radial growth / pointer years / beech / Vosges

\section{INTRODUCTION}

C'est au début des années 1980, et plus précisément en 1983, que les forestiers français ont observé, en particulier dans les Vosges (Landmann et al, 1987), l'amorce de ce qui a été appelé par la suite le dépérissement des forêts. L'alarme avait d'ailleurs déjà été donnée par les forestiers allemands qui avaient observé un dépérissement sur le sapin dans le début des années 1970, dépérissement qui a affecté par la suite l'épicéa. Ce n'était pas la première fois qu'un tel dépérissement avait été observé : au début des années 1920, le sapin avait été affecté, et ce phénomène avait été attribué à l'apparition de sécheresses importantes (König, 1979).

L'ampleur atteinte par ce dépérissement dans les années 1980 a amené scientifiques et gestionnaires à s'interroger sur le rôle possible de la pollution atmosphérique dans ce phénomène. En France, cela s'est traduit par la mise en place du programme "DEFORPA" (dépérissement des forêts attribué à la pollution atmosphérique), programme pluridisciplinaire d'étude des causes du dépérissement (Bonneau, 1987).

Dans le cadre de ce programme, une première étude dendroécologique de la sapi- nière vosgienne a été engagée à partir de 1983 (Becker, 1987). Parmi les résultats obtenus, 2 ont surpris : la tendance à l'augmentation à long terme (le siècle) de la productivité de la sapinière vosgienne, et le décalage très net dans le temps entre l'apparition du symptôme de dépérissement (défoliation) et la perte de vitalité des arbres appréciée par la largeur des cernes.

Les résultats obtenus sur le sapin dans les Vosges ont été ultérieurement confirmés dans le Jura (Bert, 1988; Bert et Becker, 1990). Parallèlement, une étude analogue était engagée sur le hêtre et les chênes en forêt de plaine (Nieminen, 1988) : les premiers résultats semblaient montrer que l'on ne retrouvait pas, chez ces feuillus, la tendance à long terme observée chez le sapin.

D'où la question : cette tendance à l'augmentation de la croissance radiale observée sur le sapin dans les Vosges, tendance que l'on ne retrouve pas avec le hêtre en plaine lorraine, est-elle propre à l'espèce sapin ou attribuable aux conditions de milieu de la montagne vosgienne?

C'est pour apporter une première réponse à cette question que nous avons décidé de lancer une étude dendroécologique du hêtre dans les Vosges. 


\section{ZONE D'ÉTUDE - ÉCHANTILLONNAGE}

Le massif vosgien étant orienté sensiblement nord-sud, on peut penser a priori que les conditions climatiques, en particulier pluviométriques, sont suffisamment différentes sur le versant lorrain et sur le versant alsacien pour justifier une étude séparée de ces 2 versants. Nous présentons ici les premiers résultats obtenus sur 85 placettes installées sur le versant lorrain, choisies de telle sorte que l'échantillon soit écologiquement aussi proche que possible de celui utilisé dans l'étude sur le sapin. Dans un premier temps, nous avons choisi de privilégier le nombre de placettes inventoriées par rapport au nombre d'arbres par placette pour gommer, dans les phénomènes observés, la part de variabilité éventuelle due à la sylviculture. Par ailleurs, il nous semble essentiel de souligner que, à ce stade de notre travail, nous n'avions pas pour objectif de mettre en évidence les causes écologiques de ces phénomènes.

En fait, dans la hêtraie-sapinière du versant lorrain, les peuplements de hêtre sont de faible superficie et très disséminés : il s'agit le plus souvent de bouquets de quelques ares, beaucoup plus rarement quelques hectares. Leur repérage directement sur le terrain aurait probablement été très long et très difficile. C'est pourquoi nous avons choisi de les repérer sur photographies aériennes: en effet, il est assez facile sur ces photographies de distinguer les peuplements feuillus des peuplements résineux, un peu plus difficile de différencier les peuplements adultes de chêne et de hêtre, parfois très difficile de distinguer un accru de lisière d'un jeune peuplement de hêtre. Les surfaces forestières interprétées comme étant constituées de hêtre ont été reportées sur des cartes IGN au 1/25000. Ce sont ces cartes qui ont été utilisées pour nous guider dans les prospections sur le terrain : il s'est avéré par la suite que cette reconnaissance préalable sur photographie aérienne nous a fait gagner un temps considérable sur le terrain.

L'aire couverte par ces 85 placettes (fig 1) va de Saverne au nord à Cornimont au sud : l'altitude varie de 320 à $1100 \mathrm{~m}$ (moyenne : $642 \mathrm{~m}$ ). Elle recouvre globalement la zone prospectée pour le sapin, à l'exception de certains secteurs (bassin de Saint-Dié) où le hêtre est pratiquement absent. Les placettes ont été choisies de façon à disposer d'un maximum de variabilité en ce qui concerne l'âge du peuplement et la situation écologique (exposition, altitude, type stationnel). Seuls ont été conservés les peuplements purs ou quasiment purs de hêtre : les peuplements en mélange pied à pied sapin-hêtre ont été rejetés ainsi que ceux présentant une très forte hétérogénéité des diamètres, laissant supposer une dynamique de la concurrence inter- ou intra-spécifique particulière, non représentative de la sylviculture "moyenne" du hêtre dans les Vosges.

Sur chaque placette, nous avons choisi 4 à 8 arbres parmi les dominants ou co-dominants, sans tenir compte de leur circonférence, mais en éliminant les sujets mal conformés (fourche basse, blessure importante...). Les principales caractéristiques de la station (altitude, exposition, position topographique, pente, type de roche mère et d'humus) ont été notées. Pour chacun des hêtres choisis, il a été prélevé à $1,30 \mathrm{~m}$ une carotte à coeur, selon une direction parallèle aux courbes de niveau (pour éviter le bois de compression). Aucune notation de l'état sanitaire des houppiers n'a, dans un premier temps, été effectuée, les tentatives d'estimation de cet état sanitaire en utilisant la méthode Roloff (in Landmann, 1988) ayant été infructueuses.

Nous avons choisi exclusivement des arbres dominants ou co-dominants pour limiter au maximum (mais non éliminer totalement) l'effet de la concurrence inter-individuelle sur la croissance en diamètre. Le statut actuel n'est en effet pas obligatoirement identique à ce qu'il a pu être dans le passé : lors de la lecture des carottes, nous avons rencontré un certain nombre de cas où des individus aujourd'hui dominants présentaient des séries de cernes extrêmement minces, laissant supposer qu'ils avaient été fortement concurrencés (dominés?) dans le passé.

Au total, 537 hêtres ont été carottés sur les 85 placettes. Après planage des carottes (on enlève, avec une lame de cutter, à peu près le tiers de l'épaisseur de la carotte, dans un plan perpendiculaire au fil du bois), les largeurs de cernes sont saisies manuellement au moyen d'une chaîne informatisée. La précision théorique de la mesure est du $1 / 100$ de $\mathrm{mm}$ : pour diverses raisons, liées principalement à la structure du bois de hêtre, nous avons vérifié qu'en pratique les mesures sont faites à + ou $-5 / 100 \mathrm{~mm}$.

\section{MÉTHODES}

\section{Les années caractéristiques}

Toutes les carottes mesurées sont interdatées selon la méthode déjà utilisée par Becker (1987) 


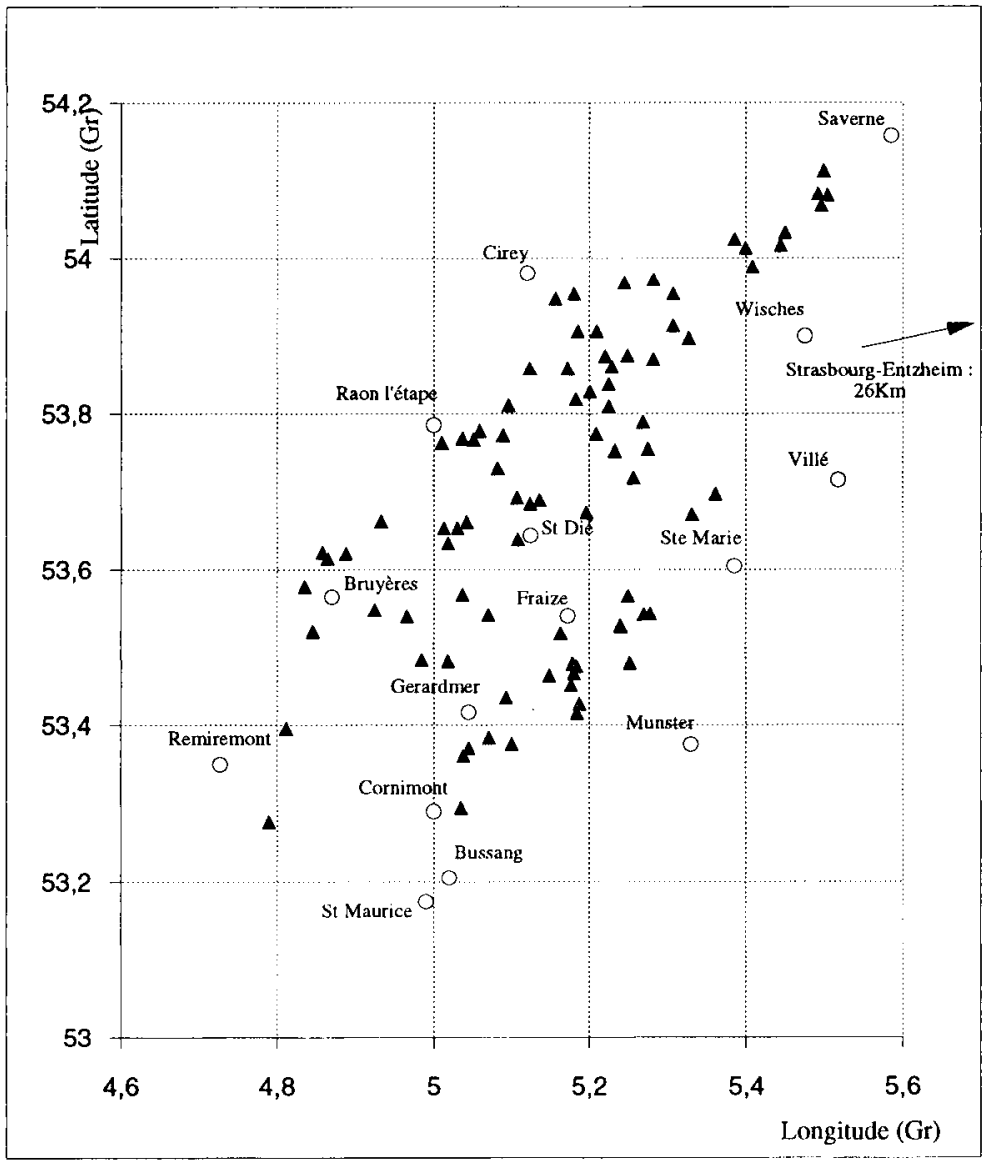

Fig 1. Localisation des placettes 1 à 85 .

et Bert (1988) : cette méthode utilise une courbe de référence (courbe moyenne de l'échantillon) ainsi que des années caractéristiques (Schweingruber et al, 1989 ; Schweingruber et al, 1991). Dans cette étude, nous avons choisi de considérer comme "caractéristique" une année quand, par rapport au cerne de l'année précédente, le cerne formé cette année-là est d'au moins $10 \%$ plus large (ou plus étroit) pour au moins $65 \%$ des arbres vivant alors ; elle est dite "très caractéristique" si plus de $75 \%$ des arbres réagissent de la même façon.

L'interdatation permet de déceler l'existence d'erreurs de saisie ou encore celle de cernes manquants. Une fois cette opération terminée, on a la quasi-certitude que chaque cerne s'est bien vu attribué sa date de mise en place.

\section{Largeur du cerne à un âge constant}

L'étude de l'évolution de la largeur des cernes à âge constant est, avec celle des indices de croissance, l'une des méthodes qui permettent de se dégager de l'influence de l'âge quand on cherche à étudier l'influence de la date sur l'évolution de la largeur des cernes. À titre d'exemple, la figure 2 illustre cette évolution pour les cernes de 30,60 et 90 ans (dans chaque cas, à plus ou moins 2 ans, pour amortir l'effet de certaines années trop 
particulières). Seuls ont été conservés les points correspondant à la moyenne d'au moins 4 arbres.

\section{Accroissement moyen pondéré selon l'âge}

L'épaisseur des cernes ayant tendance à diminuer avec l'âge (Fritts, 1976), on doit en tenir compte pour analyser l'évolution de cette largeur avec la date. Une pondération à été éffectuée pour tenir compte du fait que, pour un âge déterminé, les effectifs sont variables selon la date : on dispose de nombreuses largeurs de cernes pour les âges moyens (quelle que soit la date), mais, par exemple, plus la date est ancienne, et moins on dispose de cernes d'âge élevé, la plupart des arbres correspondants ayant déjà été exploités. De la même façon, quand on se rapproche de la date actuelle, le nombre des cernes d'âge faible diminue. La pondération introduite a essentiellement pour but de corriger, dans la courbe d'accroissement moyen en fonction de l'âge, le poids des couples "âge du cerne-date de formation» en fonction du nombre de cernes disponibles pour cet âge.

Le coefficient de pondération appliqué à un cerne donné, formé à un âge cambial a et à une date $d$, est égal au rapport $y / x$, dans lequel $x$ est le nombre de cernes formés à l'âge a et à la date $d$; y est le nombre moyen de cernes disponibles pour le même âge courant $a$, aux diverses dates pour lesquelles l'effectif de cernes est non nul.

La courbe moyenne obtenue exprime la largeur des cernes en fonction de l'âge des arbres en ayant minimisé au mieux l'effet de la date (parce que nous avons beaucoup d'arbres ayant le même âge à des dates différentes) et l'effet station (parce que nous avons beaucoup d'arbres ayant le même âge sur des stations différentes). Ceci explique que la courbe obtenue est déjà très lissée. Elle est alors ajustée grâce à 3 modèles mathématiques faisant intervenir l'âge : polynôme du $3^{e}$ degré pour la première partie de la courbe, du $5^{\text {e }}$ degré pour la deuxième partie, fonction exponentielle à argument négatif pour la troisième et dernière partie.

\section{Indice de croissance selon la date}

La courbe de l'indice de croissance en fonction de la date est obtenue en faisant, pour chaque cerne, le rapport entre la valeur mesurée sur la carotte et la valeur obtenue, au même âge, sur la courbe d'accroissement moyen pondéré en fonction de l'âge. En faisant la moyenne de tous ces indices pour une même date, on obtient, pour chaque année calendaire, un indice de croissance $I_{c}$ dégagé de l'influence de l'âge.

\section{RÉSULTATS ET DISCUSSION}

L'étude des accroissements radiaux des 537 arbres que nous avons carottés faisant appel aux mêmes méthodes que celles utilisées pour le sapin, nous comparerons nos résultats à ceux obtenus pour le sapin sur l'ensemble du massif vosgien (Becker, 1990), le cas échéant à ceux obtenus dans le Jura (Bert, 1988).

\section{Les années caractéristiques et les cernes manquants}

Le tableau I présente les différentes «années caractéristiques».

On peut remarquer que :

- les années caractéristiques avec un cerne plus large $(n=13)$ sont à peine plus nombreuses que celles à cernes plus mince $(n=$ 12) ;

- plus de la moitié de ces années caractéristiques sont postérieures à 1940, et donc relativement récentes;

- les années caractéristiques sont très peu nombreuses entre 1890 et 1940 .

La comparaison de nos années caractéristiques avec celles d'autres auteurs, et relatives à d'autres espèces, est intéressante. Becker et al (1990) ont publié un tableau donnant les années de fort ralentissement de la croissance pour quatre espèces : sapin, épicéa, hêtre et pin sylvestre, en Allemagne, Suisse et France. On y constate que certaines années sont communes aux différentes espèces (1948 


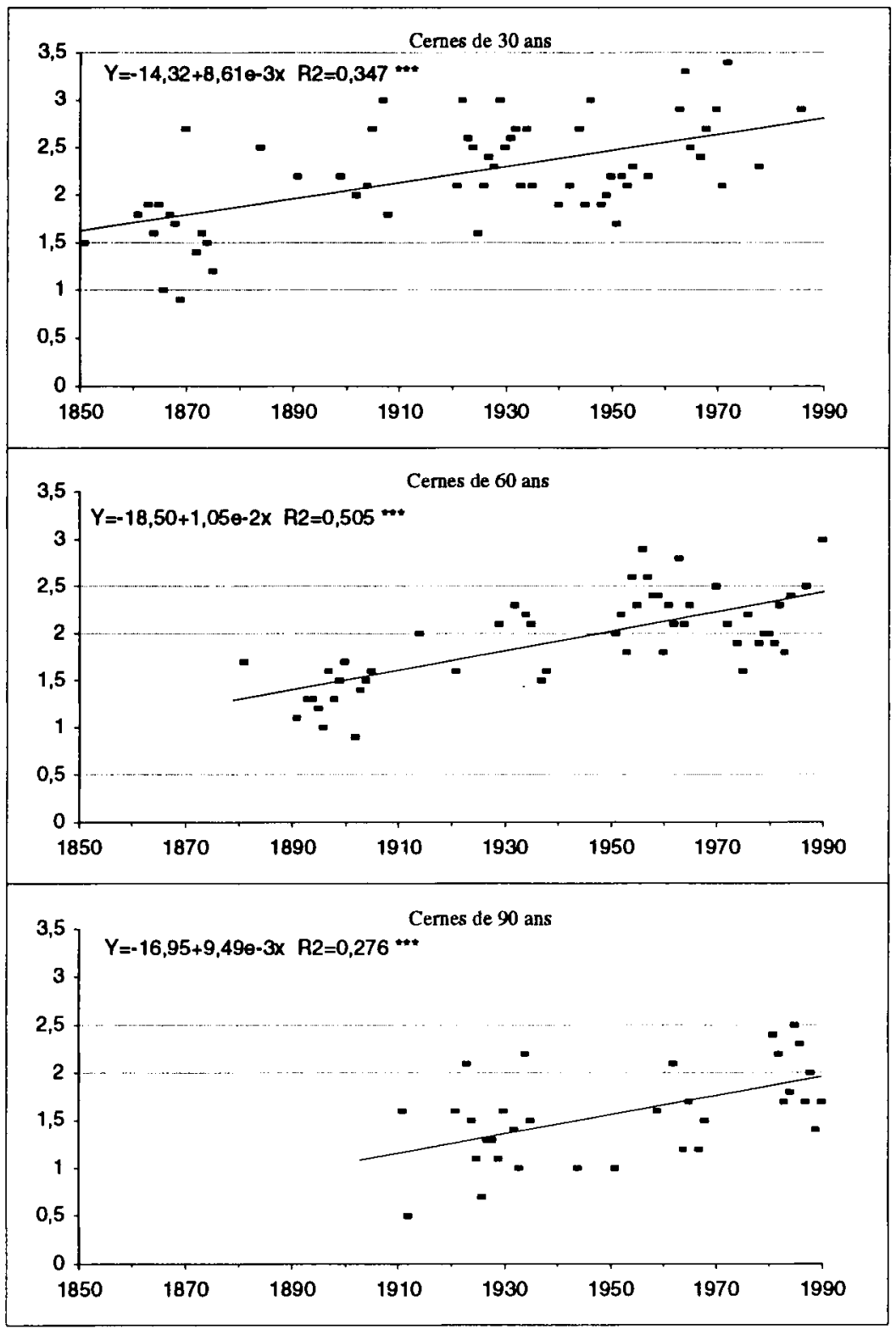

Fig 2. Évolution de la largeur de cerne au même âge $(30,60$ et 90 ans), à différentes dates $\left(^{\star \star \star}:\right.$ significatif au seuil de $1 / 1000)$.

ou 1976), d'autres paraissent spécifiques (1920 pour l'épicéa, 1956 pour le sapin). En ce qui concerne notre échantillon, nous avons retrouvé des années particulièrement défavorables comme 1934, 1945 (qui paraît d'ailleurs très spécifique à l'espèce hêtre), 1948, 1959, 1976. Parmi les années favorables, certaines sont également citées par Schweingruber et al (1991) : 1946 et 1955 en particulier. D'autres (1958, 1967 , 
1951, 1910) sont spécifiques à notre échantillon.

Contrairement au sapin dans les Vosges (Becker, 1987), les cernes manquants semblent relativement rares chez le hêtre (en tout, 41 cernes manquants sur 21 arbres, soit $4 \%$ des arbres échantillonnés). Et surtout, nous n'avons qu'exceptionnellement enregistré des cernes manquants sur une longue période ( 3 fois 3 cernes manquants ou plus). Enfin, on peut remarquer que les cernes manquants se rencontrent surtout dans les conditions difficiles des placettes d'altitude : 11 des 21 arbres qui en présentent sont situés à plus de $900 \mathrm{~m}$. En revanche, il peut arriver assez fréquemment que des limites entre cernes soient très peu visibles, ne permettant pas a priori d'identifier un cerne à cet endroit de la carotte.

La fréquence des cernes manquants sur les carottes des hêtres des Vosges paraît en revanche plus proche de celle observée sur le sapin dans le Jura (Bert, 1992). Bert, comme Elling (1987) ou encore Watzing et Fischer (1987), a montré qu'il existait un parallèle entre la fréquence des cernes manquants et l'intensité du dépérissement : ce qui est cohérent avec le fait que, toutes choses égales par ailleurs, dans les Vosges, le hêtre, essence caducifoliée moins sensible, est moins dépérissant que le sapin.

\section{Largeur du cerne à un âge constant}

On voit (fig 2) que non seulement il n'y a pas de réduction de la largeur d'un cerne de même âge entre le $x \mid x^{e}$ et le $x x^{e}$ siècle, mais encore que, quel que soit l'âge, cette largeur de cerne montre une tendance certaine à l'augmentation d'un siècle sur l'autre. Comme pour le sapin (Becker, 1987), il n'y aurait pas de réduction de la croissance depuis le milieu de siècle dernier, mais plutôt une tendance à l'augmentation.

Par ailleurs, ces graphiques confirment la diminution, en valeur absolue, de la largeur
Tableau I. Années caractéristiques (versant lorrain, 85 placettes).

$\begin{array}{lrrrr}\text { Année } & \% \text { en }+ & \% \text { en } & \%= & \text { Nb arbres } \\ & & & & \\ & & & & \\ 1945^{\mathrm{a}} & 3 & 87 & 10 & 500 \\ 1889^{\mathrm{b}} & 84 & 6 & 10 & 223 \\ 1976^{\mathrm{a}} & 7 & 82 & 11 & 537 \\ 1982^{\mathrm{b}} & 79 & 5 & 16 & 537 \\ 1859^{\mathrm{b}} & 78 & 5 & 17 & 147 \\ 1958^{\mathrm{b}} & 79 & 6 & 15 & 533 \\ 1983^{\mathrm{a}} & 6 & 76 & 18 & 537 \\ 1885^{\mathrm{a}} & 9 & 76 & 15 & 215 \\ 1934^{\mathrm{a}} & 7 & 74 & 19 & 451 \\ 1967^{\mathrm{b}} & 71 & 7 & 22 & 537 \\ 1870^{\mathrm{a}} & 11 & 75 & 14 & 164 \\ 1989^{\mathrm{a}} & 8 & 71 & 21 & 537 \\ 1858^{\mathrm{a}} & 10 & 73 & 17 & 144 \\ 1948^{\mathrm{a}} & 12 & 74 & 14 & 509 \\ 1910^{\mathrm{b}} & 70 & 8 & 22 & 334 \\ 1951^{\mathrm{b}} & 68 & 8 & 24 & 518 \\ 1955^{\mathrm{b}} & 66 & 7 & 27 & 524 \\ 1959^{\mathrm{a}} & 10 & 69 & 21 & 535 \\ 1865^{\mathrm{a}} & 11 & 69 & 20 & 157 \\ 1846^{\mathrm{b}} & 71 & 14 & 15 & 109 \\ 1931^{\mathrm{b}} & 65 & 9 & 26 & 439 \\ 1888^{\mathrm{a}} & 14 & 69 & 17 & 219 \\ 1845^{\mathrm{b}} & 69 & 14 & 17 & 105 \\ 1954^{\mathrm{b}} & 65 & 13 & 22 & 524 \\ 1946^{\mathrm{b}} & 66 & 19 & 15 & 506\end{array}$

a Années où le cerne est plus étroit que l'année précédente; ${ }^{b}$ années où le cerne est plus large que l'année précédente. Les colonnes $\%$ indiquent la proportion d'arbres ayant le cerne de l'année plus large, moins large ou égal à celui de l'année précédente.

des cernes avec l'âge: vers 1990, les cernes élaborés à 30 ans sont près d'une fois et demie plus larges que ceux élaborés à 90 ans.

\section{Accroissement moyen pondéré selon l'âge}

La courbe de la figure 3 représente "l'accroissement moyen pondéré" selon l'âge courant. Elle peut être schématiquement divisée en 3 parties : 
- de 0 à 10 ans environ, une augmentation forte et rapide de la largeur du cerne qui atteint près de $2 \mathrm{~mm}$ à 10 ans ;

- de 11 à 44 ans, une augmentation encore sensible, mais moins régulière, de la largeur des cernes qui dépasse légèrement $2 \mathrm{~mm}$;

- de 45 à 140 ans, une diminution assez lente, diminution qui se ralentit avec l'âge. L'extrapolation de l'ajustement donne une largeur de cerne d'environ $1,2 \mathrm{~mm}$ à 150 ans (l'allure de la courbe paraît anormale à partir de 140 ans, très probablement en raison des effectifs qui chutent de façon très nette à partir de cet âge : nous n'avons donc pas tenu compte de la remontée de la courbe et utilisé la même équation à partir de 45 ans).

Cette courbe d'accroissement moyen pondéré du hêtre est assez différente de celle du sapin dans les Vosges, plus proche de celle du sapin du Jura : alors que le sapin, dans les Vosges (Becker, 1987), présente un maximum de croissance avant 10 ans, ce même sapin, dans le Jura, présente un plateau entre 10 et 40 ans, comme le hêtre dans les Vosges. D'abord interprété (Bert et Becker, 1990) comme un effet de la concurrence des peuplements adultes sur la régénération naturelle, ce plateau sera inter- prété ultérieurement (Bert, 1992) comme le reflet d'une lente évolution du potentiel de croissance juvénile dont le maximum se déplace, depuis le siècle dernier, vers des âges de plus en plus élevés. Nous ne disposons pas encore d'un échantillon suffisant pour conclure en ce qui concerne le hêtre, mais la deuxième explication avancée par Bert paraît plus vraisemblable dans notre cas, dans la mesure où nous n'avons que des peuplements de futaie régulière : il est peu probable dans ces conditions qu'il y ait un effet du traitement sylvicole qui a dû être relativement stable (dans l'espace et dans le temps) sur le versant lorrain des Vosges. II n'est en revanche pas impossible que certains peuplements de très faible superficie aient eu à souffrir, dans leur jeunesse, de la concurrence des peuplements de sapin voisins.

On observe par ailleurs que :

- chez le sapin des Vosges, la diminution de la largeur du cerne est forte et assez rapide jusqu'à 80 ans; chez le sapin du Jura, elle est moins forte et se poursuit jusque vers 90 ans ; pour le hêtre, elle est également moins forte et se stabilise vers 90 ans ;

- en valeur absolue, le ralentissement de croissance du sapin est beaucoup plus

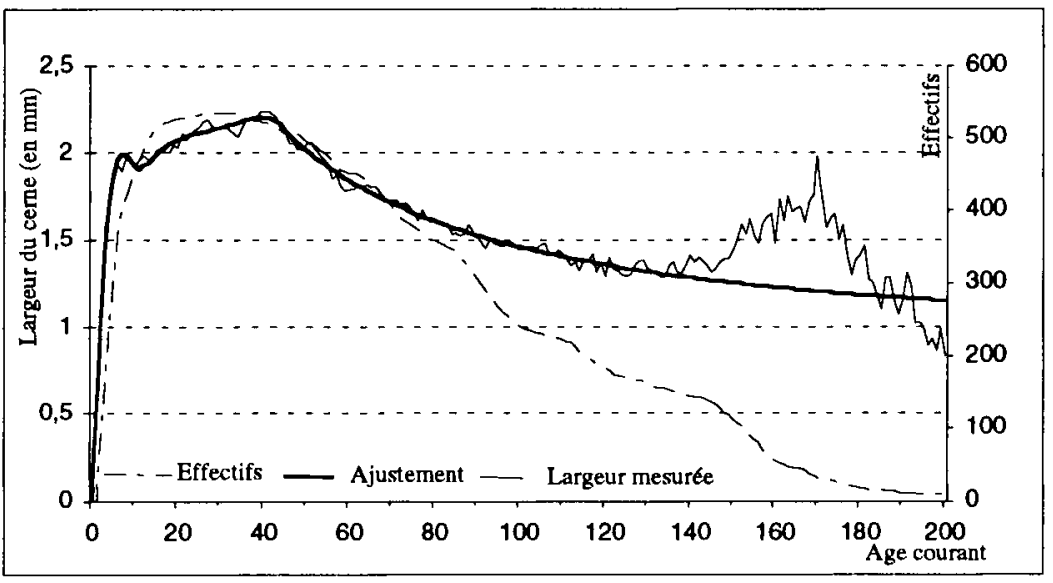

Fig 3. Accroissement moyen selon l'âge courant, ajustement et effectifs des hêtres des 85 placettes du versant lorrain des Vosges. 
important que celui du hêtre : à 100 ans, les sapins des Vosges font (en moyenne) des cernes de $1 \mathrm{~mm}$, et les hêtres des cernes de $1,4 \mathrm{~mm}$ (ceux des sapins du Jura sont de $1,5 \mathrm{~mm}$ ).

\section{Indice de croissance selon la date}

L'étude de cette courbe (fig 4) peut être faite selon 3 niveaux d'approche : global, à moyenne fréquence et à haute fréquence.

Globalement, cette courbe montre une nette tendance à long terme à l'augmentation de la largeur des cernes du hêtre : entre 1850 et 1989 , cette augmentation est de l'ordre de $70 \%$, du même ordre de grandeur que pour le sapin.

Mais l'allure générale de la courbe obtenue pour le hêtre diffère sensiblement de celles obtenues pour le sapin pour lequel, dans les Vosges comme dans le Jura, l'indice de croissance présente une pente moyenne assez forte et régulière entre 1850 et 1930. À partir des années trente, on observe un palier net : depuis cette période, il n'y a pratiquement pas eu de gain de croissance sur le diamètre, et même, dans les Vosges, on observe une baisse sensible de l'indice, qui passe de $110-120 \%$ à $100-$ $110 \%$. Chez le hêtre, on ne retrouve pas ce palier, et le gain de croissance (si l'on excepte certains épisodes difficiles) est à peu près constant depuis 1850 .

Différentes hypothèses ont été jusqu'ici avancées pour expliquer la tendance à long terme à l'augmentation de la largeur des cernes. Les facteurs écologiques les plus fréquemment évoqués sont le climat (température et pluviométrie), l'augmentation du taux de $\mathrm{CO}_{2}$ (par ses effets directs sur la photosynthèse, mais aussi indirects sur la pluviométrie et les températures : Becker, 1990), plus rarement et probablement de façon moins générale, les dépôts azotés atmosphériques (Bert, 1992).

À moyenne fréquence, cette courbe montre l'existence de dépressions (ralentissements de la croissance ou "crises") plus ou moins profondes et longues. Deux de ces crises paraissent particulièrement fortes : celle de 1863-1879 qui a atteint son paroxysme en 1870, et celle de 1939-1955 qui l'a atteint en 1948. D'autres crises, de plus faible importance (dans leur durée comme dans leur amplitude), sont également visibles en 1884-1989 (minimum en 1888), 1916-1924 (minimum en 1922) et 1975-1980 (minimum en 1976).

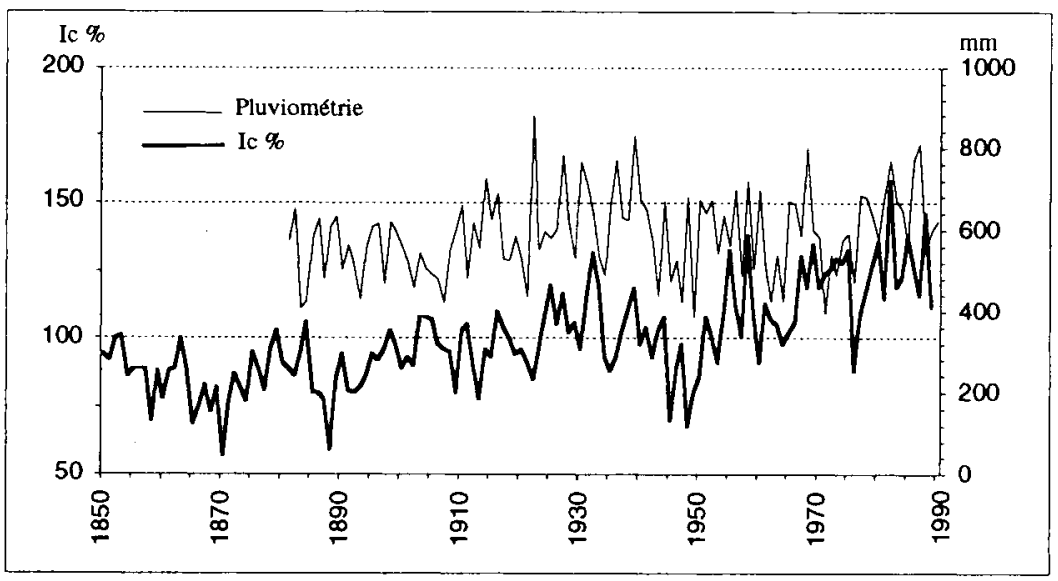

Fig 4. Indice de croissance moyen selon la date du hêtre sur le versant lorrain des Vosges (85 placettes) et pluviomé $t r i e$ annuelle à Strasbourg. 
II existe une parenté certaine avec les périodes de crise mises en évidence pour le sapin dans les Vosges et dans le Jura. La différence réside surtout dans l'intensité et la durée de ces crises, qui ne sont pas strictement identiques pour les espèces et les régions d'étude.

La courbe de pluviométrie de Strasbourg (station météorologique qui est à la fois la plus proche et pour laquelle on dispose des relevés les plus anciens) montre (fig 4), aux variations de haute fréquence près, une évolution à peu près parallèle à celle de l'indice de croissance : augmentation de 1881 (date des premiers relevés) à 1930, diminution entre 1930 et 1950 , reprise après 1970 (normale 1881-1910: $521 \mathrm{~mm}$; normale 1961-1990:606 mm). À partir de 1970 , on observe un décalage entre les 2 courbes : pendant plusieurs années, le hêtre maintient un niveau de croissance élevé alors que le déficit pluviométrique n'a jamais été aussi important depuis le début des relevés météorologiques. Une analyse plus fine du déterminisme climatique des variations de la croissance radiale du hêtre sera faite ultérieurement.

\section{CONCLUSION}

Cette étude des accroissements radiaux moyens des hêtres des 85 placettes du versant lorrain des Vosges nous amène à proposer provisoirement les conclusions suivantes.

L'accroissement moyen selon l'âge courant suit une loi assez différente chez le hêtre et chez le sapin. Le sapin des Vosges présente un accroissement maximum vers 10 ans (près de $3 \mathrm{~mm}$ ), puis la largeur des cernes diminue rapidement entre 20 et 80 ans, pour se stabiliser vers 100 ans (autour de $0,8 \mathrm{~mm}$ ). Chez le hêtre, la croissance maximum est atteinte plus tard, vers 40 ans (un peu plus de $2 \mathrm{~mm}$ ), puis elle se ralentit lentement pour atteindre, vers 100 ans, environ $1,4 \mathrm{~mm}$.

La courbe d'indice de croissance du hêtre selon la date évolue de façon sensiblement différente de celle du sapin : la croissance du sapin est caractérisée par une augmentation assez forte et régulière entre 1860 et 1930 , puis un palier net à partir de cette date; pour le hêtre, le gain de croissance paraît, à quelques épisodes difficiles près, à peu près continu depuis 1850 .

Contrairement au sapin dans la même aire d'étude, où il a été mis en évidence un problème fréquent de cernes manquants, ce phénomène est relativement rare chez le hêtre.

Ceci nous conduit à penser que le comportement du hêtre est sensiblement différent de celui du sapin dans les Vosges. Cet effet «espèce» n'est d'ailleurs pas nouveau : Borel et Serre (1969) l'avaient déjà montré dans leur étude sur les hêtraies sapinières du sud de la France : les hêtres seraient surtout sensibles aux précipitations de l'automne précédent, alors que les sapins, dans la même situation, y sont beaucoup moins sensibles; en revanche, ils seraient plus sensibles aux basses températures.

Mais l'effet espèce n'exclut pas un effet milieu que l'absence de tendance à long terme trouvée sur le plateau lorrain par Nieminen laisse supposer. D'autre part, on constate, à propos des années caractéristiques, qu'elles sont loin d'être les mêmes dans les Vosges et dans la plaine lorraine: 1954, année favorable dans les Vosges, 1945 et 1948, années défavorables, ne sont pas caractéristiques à Amance (1945 serait plutôt une année favorable...).

Nous n'en sommes qu'à la première phase de notre étude : il nous a cependant semblé intéressant, sur cet échantillon "restreint", d'établir la tendance moyenne à long terme de l'augmentation de la largeur des cernes chez le hêtre. Ce résultat vient 
conforter ceux obtenus précédemment dans notre laboratoire.

Il sera intéressant d'essayer de comprendre, voire d'expliquer, sur un échantillon de placettes largement complété (pour «amortir» le «bruit» éventuel lié au passé sylvicole), les différences dont nous avons pu faire état avec le sapin, dans ce même massif des Vosges, et analyser finement l'incidence sur la croissance du hêtre des différents facteurs écologiques auxquels la hêtraie vosgienne est soumise.

L'auteur remercie l'échelon nancéen de I'Inventaire forestier national (IFN) qui a mis à sa disposition les photographies aériennes des Vosges, ainsi que les techniciens du laboratoire de phyto-écologie pour leur participation active à la phase de terrain de l'enquête, et tout particulièrement MM C Kieffer, F Gérémia et R Schipfer.

\section{RÉFÉRENCES}

Becker M (1987) Bilan de santé actuel et rétrospectif du sapin (Abies alba Mill) dans les Vosges. Étude écologique et dendrochronologique. Ann Sci For 44 , 4, 379-402

Becker M, Landmann G, Levy G (1989) Silver fir decline in the Vosges mountains (France): role of climate and silviculture. Water Air Soil Pollut 48, 77-86

Becker M, Bräker OU, Kenk G, Schneider O, Schweingruber FH (1990) Aspect des houppiers et croissance des arbres au cours des dernières décennies dans les régions frontalières d'Allemagne, de France et de Suisse. Rev For Fr XLII 3, 284-300

Becker M (1990) Sensibilité des écosystèmes forestiers aux facteurs climatiques et aux pratiques sylvicoles. Approche dendroécologique. In : Le dépérissement des érablières, causes et solutions possibles ( $C$ Camiré, W Hendershot, D Lachance, eds), CRBF, Fac For Géom, Univ Laval, Québec, Canada 9-19

Bert GD (1988) Étude dendroécologique du dépérissement du sapin (Abies alba Mill) dans le Jura. Mémoire de DEA, Université de Nancy I, $60 \mathrm{p}$

Bert GD (1992) Influence du climat, des facteurs stationnels et de la pollution sur la croissance et l'état sanitaire du sapin pectiné (Abies alba Mill) dans le Jura. Étude phytoécologique et dendrochronologique. Thèse de doctorat d'Université, Nancy I, 200 $p+$ annexes

Bert GD, Becker M (1990) Vitalité actuelle et passée du sapin (Abies alba Mill) dans le Jura. Étude dendroécologique. Ann Sci For 47, 395-412

Bonneau M (1987) Genèse et contenu du programme Deforpa. In : Les recherches en France sur le dépérissement des forêts. Programme Deforpa, 1 er rapport, ENGREF-Nancy, 6-10

Borel L, Serre F (1969) Phytosociologie et analyse des cernes ligneux : l'exemple de 3 forêts du haut Var. Oecol plant IV, 155-176

Fourchy P (1951) Secheresse, variations climatiques et végétation. Rev For Fr III 1, 47-55

Fritts HC (1976) Tree-ring and climate. Academic Press, New York, États-Unis, $567 \mathrm{p}$

von Elling W (1987) Eine Methode zur Erfassung von Verlauf und Grad der Schädingung von Nadelbaumbeständen. Eur J For Pathol 17, 426-440

König E (1979) Entwicklungstendenzen bei der Tannenerkrankung. Forst-Holzwirt 16, 361-366

Landmann G (1988) Comment apprécier la vitalité d'un arbre ou d'un peuplement forestier? Rev For Fr XL, 4, 265-284

Landmann G (1991) Observation au sol de l'état sanitaire des forêts. In : Les recherches en France sur le dépérissement des forêts (G Landmann, ed), Programme Deforpa, $2^{\circ}$ rapport, ENGREF-Nancy, 723

Landmann G, Bonneau M, Adrian M (1987) Le dépérissement du sapin pectiné et de l'épicéa commun dans le massif vosgien est-il en relation avec l'état nutritionnel des peuplements ? Rev For Fr XXXIX, 1, 5-11

Nieminen TM (1988) Étude dendroécologique du chêne (pédonculé et sessile) et du hêtre dans une forêt de la plaine lorraine (forêt domaniale d'Amance) Mémoire de DEA, Université de Nancy I, $40 p+$ annexes.

Schweingruber $F H$, Eckstein D, Serre-Bachet $F$, Bräker OU (1989) Identification, presentation and interpretation of event years and pointer years in dendrochronology. Informal meeting of dendrochronologists from southern and central Europe held in Loumarin, Provence, France, on 4 May 1989, 9-39

Schweingruber FH, Wehrli U, Aellen-Rumo K, Aellen M (1991) Weiserjahre als Zeiger extremer Standortseinflüsse. Schweiz $Z$ Forstwes 142, 1, 33-52

Wätzig H, Fischer B (1987) Untersuchungen über den Jahrringausfall an Fichtenbeständen. Wiss $Z$ Tech Univ Dresden 36, 273-275 